\title{
Magnetotransport properties depending on the nanostructure of $\mathrm{Fe}_{3} \mathrm{O}_{4}$ nanowires
}

\author{
M Abid ${ }^{1}$, J-P Abid, S Jannin, S Serrano-Guisan, I Palaci and \\ J-Ph Ansermet \\ IPN/SB, Ecole Polytechnique Fédérale de Lausanne, station 3, CH-1015, Lausanne, Switzerland \\ E-mail: mabid@chimie.u-strasbg.fr
}

Received 19 March 2006, in final form 23 May 2006

Published 19 June 2006

Online at stacks.iop.org/JPhysCM/18/6085

\begin{abstract}
We have studied the magnetic behaviour of $\mathrm{Fe}_{3} \mathrm{O}_{4}$ nanowires (NWs) with two different diameter ranges, above $150 \mathrm{~nm}$ and below $60 \mathrm{~nm}$, made by electrodeposition techniques into a polymeric template. The nanowires were characterized using various techniques, in particular Mössbauer and thermoelectrical power measurements. The stoichiometric distribution of $\mathrm{Fe}$ cations showed clearly the presence of the magnetite inverse spinel electronic structure. Structural analysis performed using high-resolution transmission electron microscopy revealed two kinds of nanowire morphologies depending on the size. For nanowires above $150 \mathrm{~nm}$ in diameter, a contiguous network of well-bound nanoparticles was obtained. Instead, with a diameter of $60 \mathrm{~nm}$, a polycrystalline structure was observed. The largest nanowires presented a magnetoresistance (MR) greater than $10 \%$, whereas the thinner nanowires had almost none.
\end{abstract}

\section{Introduction}

In the last decade considerable interest has developed in all effects related to spin-dependent transport like giant magnetoresistance (GMR) [1, 2] and tunnelling magnetoresistance (TMR) [3]. Hence, the half-metallic character of ferromagnetic oxide, in particular magnetite [4], nanomaterials appears to be quite promising for applications in spintronics. This theoretically perfect spin-polarization, together with its relatively high Curie temperature, might be exploited in a variety of advanced devices such as highly sensitive magnetic sensors (e.g. read heads for magnetic recording). Magnetite should considerably enhance the efficiency of these devices, in which the current spin-polarizing materials can only achieve about $40 \%$. Non-perfect magnetite crystals have the particularity of being composed of small islands of stoechiometrically perfect crystallinity separated by anti-phase boundaries (APB) [5, 6].

1 Author to whom any correspondence should be addressed. 
These APBs result from shifts or rotations of the iron sub-lattices relative to the crystal lattice, and the magnetite islands couple either ferromagnetically or anti-ferromagnetically. This can be observed experimentally as a magnetoresistive GMR-like response [5], in a similar way to the magnetoresistance of multilayers made of magnetic and non-magnetic materials. Transfer of spin-polarised electrons between grain boundaries also gives rise to magnetoresistive effects and this has been observed in compacted magnetite powders [7]. Magnetite is also characterized by metal to insulator transition (more precisely, semiconductorinsulator or semiconductor-semiconductor) around $T_{\mathrm{v}}=110 \mathrm{~K}$, known as the Verwey transition, which is an ordering effect of the iron ions $\mathrm{Fe}^{2+}$ and $\mathrm{Fe}^{3+}$ within the $\mathrm{B}$ sites (octahedron sites) of the inverse spinel lattice structure. For TMR devices, the preparation of magnetite nanomaterials requires low deposition and thin film techniques. Nevertheless, the possible applications of magnetite in devices rely on the assumption that the physical properties are the same as those of bulk material. Unfortunately, the physical properties such as resistivity, magnetoresistance $[8,9]$ or magnetic behaviour of $\mathrm{Fe}_{3} \mathrm{O}_{4}$ strongly deviate from bulk properties. This has often been ascribed to the presence of anti-phase boundaries. In this framework, we have fabricated nanowires of different diameters and morphologies by electrodeposition using polymeric templates. The obtained results provide a clear picture of the dependence of the transport on the nanowires diameter size, shedding light on some aspects of magnetotransport depending of nanostructure morphology.

\section{Experimental section}

The $\mathrm{Fe}_{3} \mathrm{O}_{4}$ synthesis was achieved by means of an electroprecipitation method [10]. Typically, the electrodeposition experiment was carried out in a classical three electrode glass cell. The circuit loop was closed using a saturated $\mathrm{Ag} / \mathrm{AgCl}$ electrode as a reference and a platinum wire as a counter electrode. The potential window was estimated using cyclic voltammetry. All experiments were performed at $343 \mathrm{~K}$ under $\mathrm{N}_{2}$ bubbling during at least $1 \mathrm{~h}$ and employing the EGG 273A potentiostatic system. The morphological analysis was achieved using a Philips CM300 high-resolution transmission microscope (HRTEM) and a Philips XL30 FEG scanning electron microscope. The structural analysis of the magnetite nanomaterials was performed using a Siemens D5000 Bragg-Brentano diffractometer operating in the $\theta-2 \theta$ Bragg configuration. The voltage was set at $50 \mathrm{kV}$ with a $45 \mathrm{~mA}$ flux. The electrical transport and magnetization properties were performed using nanowires typically $6 \mu \mathrm{m}$ long with a diameter of $60 \mathrm{~nm}$ or above $150 \mathrm{~nm}$. During the electrodeposition procedure, a single contact was made between two gold layers sputtered on both sides of the membranes. The resistance of the contacted nanowire was measured in a magnetic field of $-8 \mathrm{kOe}<H<8 \mathrm{kOe}$ and in the temperature range of 70-300 K. High-precision thermoelectric power measurements by lock-in amplifier detection were carried out using a laser beam as an oscillatory source [11].

\section{Results and discussion}

\subsection{Structural properties}

Figure 1(a) shows a scanning electron micrograph of the $\mathrm{Fe}_{3} \mathrm{O}_{4}$ nanowires after removing the polycarbonate. The nanowires are about $150 \mathrm{~nm}$ in size with a density of $10^{9} \mathrm{~cm}^{-2}$. As portrayed in figure 1(b), energy-dispersive $\mathrm{x}$-ray analysis confirms the magnetite stoichiometry $\left(\mathrm{Fe}_{3} \mathrm{O}_{4}\right)$ with an average atomic ratio of $\mathrm{O} / \mathrm{Fe} 1.33$. The bright-field transmission micrographs display $\mathrm{Fe}_{3} \mathrm{O}_{4}$ nanowires with a diameter above $150 \mathrm{~nm}$ (figure 1(c)) and of $60 \mathrm{~nm}$ (figure 1(d)). The TEM analysis reveals that the as-prepared nanowires above $150 \mathrm{~nm}$ are made with 


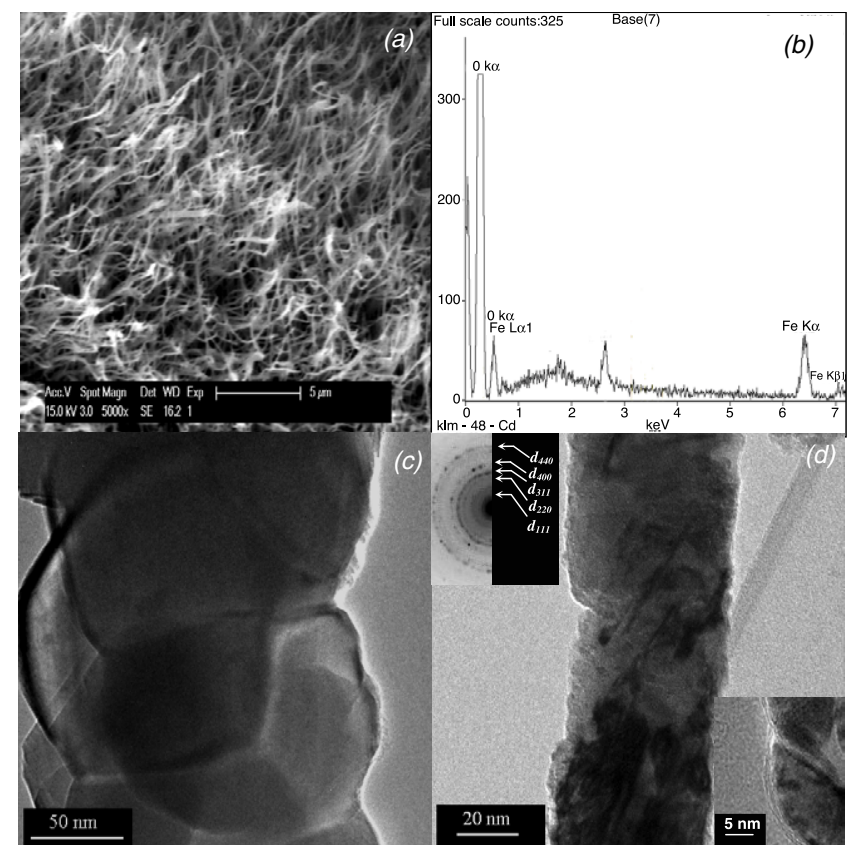

Figure 1. (a) SEM picture after removing the polycarbonate membrane, (b) EDS spectra, (c) TEM micrographs of NWs above $150 \mathrm{~nm}$ and (d) around $60 \mathrm{~nm}$ (inset shows: (left) the SAED (selected area diffraction); (right) the HRTEM micrograph).

agglomerates of nanoparticles. As shown in figure 1(c), the large nanoparticles merge together in order to form welded-neck junctions and grain boundaries. For nanowires of smaller sizes (around $60 \mathrm{~nm}$ ), we observe granular wires containing a broad range of nanoparticle sizes (size below $10 \mathrm{~nm}$ ). However, it must be pointed out that nanoparticles do not show the typical junction or grain boundaries between the nanoparticles (inset figure 1(d)). The magnetite structural study using SAED indicates the presence of a cubic structure of $\mathrm{Fe}_{3} \mathrm{O}_{4}$ and/or $\gamma$ $\mathrm{Fe}_{2} \mathrm{O}_{3}$ (see inset of figure $1(\mathrm{~d})$ ), which is consistent with the $\mathrm{x}$-ray diffraction (XRD) result (not shown). XRD cannot be more specific because $\gamma-\mathrm{Fe}_{2} \mathrm{O}_{3}$ (maghamite) and $\mathrm{Fe}_{3} \mathrm{O}_{4}$ have similar cubic inverse spinel structure with nearly the same lattice parameter ( $a=0.8350$ and $a=0.8396 \mathrm{~nm}$, respectively). To ensure the absence of impurities in the magnetite sample at room temperature, Mössbauer measurements on $\mathrm{Fe}_{3} \mathrm{O}_{4} \mathrm{NWs}$ are performed and a typical result is portrayed in figure 2(a). The determination of stoichiometry using Mössbauer spectroscopy is based on the rapid electron hopping process at room temperature. Since this hopping process is much faster than the lifetime of the excited ${ }^{57} \mathrm{Fe}$ nucleus, the B site ions appear as one average ' $\mathrm{Fe}^{2.5+}$ '-like component [12]. Therefore, at room temperature, the Mössbauer spectrum of $\mathrm{Fe}_{3} \mathrm{O}_{4}$ will contain only two components: one originating from the A sites containing $\mathrm{Fe}^{3+}$ ions, and the other from the $\mathrm{B}$ sites containing $\mathrm{Fe}^{2.5+}$ ions. Each component is split into a sextet, due to hyperfine interactions between the nuclear magnetic dipole moment and the internal magnetic field caused by the ferrimagnetically ordered electron spins. A typical spectrum of $\mathrm{Fe}_{3} \mathrm{O}_{4}$ nanowires (figure 2(a)) is fitted with two sextet components yielding an IS value (relative to $-\mathrm{Fe}$ ) of $0.2969 \mathrm{~mm} \mathrm{~s}^{-1}$ and $\mathrm{B}=49.53(9)$ for the first sextet, and $\mathrm{IS}=0.6386(6)$ and $\mathrm{B}=46.21(6)$ for the second sextet. Our fitting parameters are in good agreement with literature reports for $\mathrm{Fe}_{3} \mathrm{O}_{4}[13,14]$. The components with the larger $\mathrm{B}$ and smaller IS corresponds to the $\mathrm{Fe}^{3+}$ component. The intensity ratio defined by $\beta=I(3+) / I(2.5+)$ of the two components 

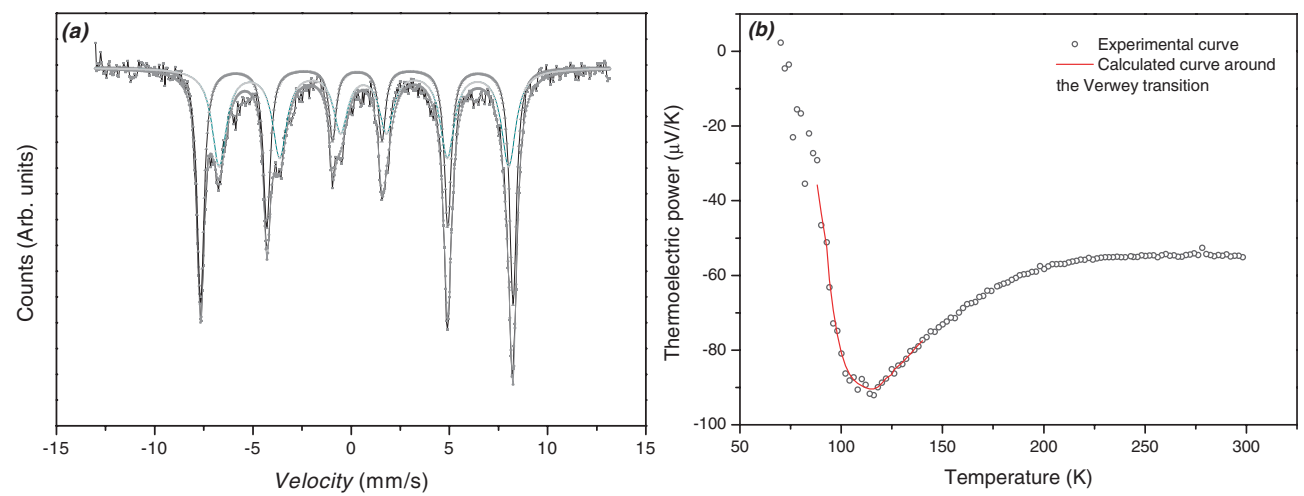

Figure 2. (a) A typical room-temperature ${ }^{57} \mathrm{Fe}$ Mössbauer spectrum of $\mathrm{Fe}_{3} \mathrm{O}_{4}$ spinel ferrite at zero field of $\mathrm{Fe}_{3} \mathrm{O}_{4}$ NWs. (b) Typical room-temperature experimental data of thermoelectrical power measurement of magnetite NWs, the calculated curves (in red) values according to the expression (2) using $2 \Delta=0.106 \mathrm{eV}$ and $q_{\mathrm{p}}-q_{\mathrm{n}}=0.01 \mathrm{eV}$.

(This figure is in colour only in the electronic version)

is a very sensitive measure of the stoichiometry [15]. This is equal to $1 / 2$ for stoichiometric $\mathrm{Fe}_{3} \mathrm{O}_{4}$, and goes to infinity for $\gamma-\mathrm{Fe}_{2} \mathrm{O}_{3}$. The non-stoichiometric value $\beta$ can be related to expression (1).

Effectively, by considering the charge neutrality, we can write non-stoichiometric $\mathrm{Fe}_{3-\alpha} \mathrm{O}_{4}$ as $\mathrm{Fe}_{\mathrm{A}}^{3+}\left[\mathrm{Fe}_{1+2 \delta}^{3+} \mathrm{Fe}_{1-3 \delta}^{2+} \square_{\delta}\right]_{\mathrm{B}} \mathrm{O}_{4}^{2-}$, where $\square$ stands for a vacancy. This leads to $\mathrm{Fe}_{\mathrm{A}}^{3+}\left[\mathrm{Fe}_{1-3 \delta}^{3+} \mathrm{Fe}_{1-3 \delta}^{2+} \mathrm{Fe}_{5 \delta}^{3+} \square\right]_{\mathrm{B}} \mathrm{O}_{4}^{2-}$, since every vacancy traps five $\mathrm{Fe}^{3+}$ ions. Including hopping, we finally obtain $\mathrm{Fe}_{\mathrm{A}}^{3+}\left[\mathrm{Fe}_{2-6 \delta}^{2.5+} \mathrm{Fe}_{5 \delta}^{3+} \square_{\delta}\right]_{\mathrm{B}} \mathrm{O}_{4}^{2-}$. So, $\beta$ is given by the relation:

$$
\beta=\frac{1+5 \delta}{2-6 \delta} \text {. }
$$

We observe a slight non-stoichiometric phase which corresponds to a composition phase $\mathrm{Fe}_{2.96} \mathrm{O}_{4}$ and $\mathrm{Fe}_{2.97} \mathrm{O}_{4}$ for $60 \mathrm{~nm}$ and above $150 \mathrm{~nm}$ diameter size nanowires, respectively.

To investigate the presence of impurities and confirm the non-stoichiometric $\mathrm{Fe}_{3} \mathrm{O}_{4}$ nanowires' character, the study of the thermoelectric properties at the Verwey transition $\left(T_{\mathrm{v}}\right)$ has been envisaged. Kuipers et al analysed thermoelectric power measurements on pure and doped magnetite crystals, and concluded that minor changes in stoichiometry have a deep influence on whether $\mathrm{p}$ - or n-type conduction is observed below $T_{\mathrm{v}}$ [17]. Figure 2(b) displays the evolution of the absolute thermoelectric power with the temperature. The thermoelectric power decreases gradually from $200 \mathrm{~K}$ and reaches a minimum at $115 \mathrm{~K}$. At lower temperatures, the thermoelectric power increases again and becomes positive, pointing to positive charged carriers. These values of Seebeck coefficient above the Verwey transition are in good agreement with the values reported in literature $[18,19]$. The thermoelectrical power results are fitted using the mixed conduction model [17]. This model consists of assuming that an energy gap exists between two kinds of electron states, localized on $\mathrm{Fe}^{2+}$ and $\mathrm{Fe}^{3+}$ ions, respectively. Thus one can distinguish two kinds of charge carriers resulting in a mixed conduction: holes in the $\mathrm{Fe}^{3+}$ levels and electrons on the $\mathrm{Fe}^{2+}$ levels. Accordingly to this model, the Seebeck coefficient can be expressed as a function of the temperature:

$$
S=\frac{1}{e T} \frac{-n(\Delta-\epsilon)+P(\Delta-\epsilon) \times \mu_{\mathrm{p}} / \mu_{\mathrm{n}}}{n+p \times \mu_{\mathrm{p}} / \mu_{\mathrm{n}}}
$$

where $\mu_{\mathrm{p}}$ and $\mu_{\mathrm{n}}$ are the mobilities of the $\mathrm{p}$ - and n-type charge carriers, respectively, which are assumed to be thermally activated, $\Delta$ represents the energy gap to create a charge carrier, and 

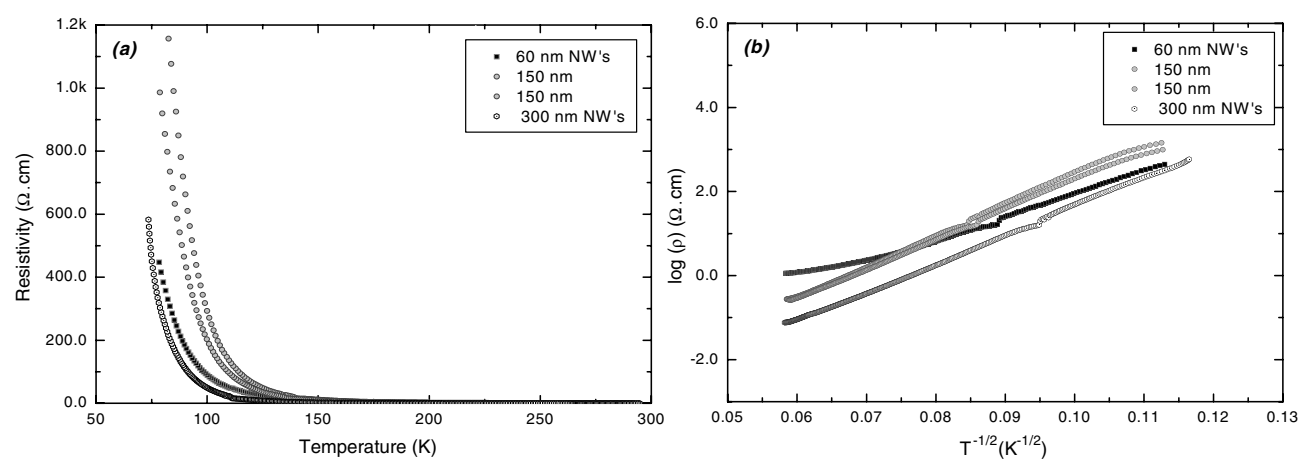

Figure 3. (a) Resistivity versus temperature with different size diameters of $\mathrm{Fe}_{3} \mathrm{O}_{4} \mathrm{NWs}$; (b) shows the resistivity plotted as $\log (\rho)$ versus $T^{-1 / 2}$.

finally $\gamma$ is the cation vacancy concentration defined by $\mathrm{Fe}_{2+2 \delta}^{3+} \mathrm{Fe}_{1-3 \delta}^{2+} \mathrm{V}_{\gamma} \mathrm{O}_{4}$. The best fitting is obtained for $q_{\mathrm{p}}-q_{\mathrm{n}}=0.01 \mathrm{eV}, 2 \Delta=0.09-0.10 \mathrm{eV}$, and values of $\gamma=0.035$ and 0.02 , which correspond to the stoichiometry of $\mathrm{Fe}_{2.96} \mathrm{O}_{4}$ for $60 \mathrm{~nm}$ and $\mathrm{Fe}_{2.98} \mathrm{O}_{4}$ for nanowires above $150 \mathrm{~nm}$, respectively. In summary, Mössbauer and thermoelectric measurements show that the $\mathrm{Fe}_{3} \mathrm{O}_{4}$ nanowires made by electrochemical methods exhibit a slight non-stoichiometric phase. We presume that this is due to the wet condition used during fabrication.

\subsection{Transport properties}

The temperature-dependent resistivity results are shown in figures 3(a) and (b). The electrical transport properties have been investigated for nanowires with different sizes. Roomtemperature resistivities of the 60,150 and $300 \mathrm{~nm}$ nanowires are $1.277,0.281$ and $0.077 \Omega \mathrm{cm}$ respectively, which are much higher than the value $(0.0004 \Omega \mathrm{cm})$ for $150 \mathrm{~nm}$ epitaxial grain boundaries [20]. Whatever the diameter size of the nanowire, the resistivity increases exponentially with decreasing temperature. These transport features on such nanostructures has the form of $\log [\rho]=T^{-1 / 2}$.

This behaviour is ascribed to a fluctuation-induced tunnelling conduction mechanism in disordered materials [21], in which the thermally activated voltage fluctuation barriers play a role in determining the temperature dependence of the conductivity. Further analysis (figure 3(b)) shows that decreasing the diameter of the nanowires induces an increase in the resistivity. Such behaviour can be understood by introducing the fact that, when the diameter of the nanowire is in the range of, or smaller than, the mean free path of the electrons, the electrical resistivity of the conductor will increase [22]. Similar behaviour has been reported on granular $\mathrm{Fe}_{3} \mathrm{O}_{4}$ thin film by Liu et al [23]. In the present case, for nanowires with a diameter of around $60 \mathrm{~nm}$, the higher resistivity observed in the temperature range from 300 to $160 \mathrm{~K}$ can be attributed to the diffusive scattering of the conduction electrons at the surface. Finally, contrary to the 'bulk' $\mathrm{Fe}_{3} \mathrm{O}_{4}$ material, no sharp Vervey transition [24] is observed. Park et al [25] suggest that the tunnelling effect due to the grain boundaries can causes the disappearance of the Verwey transition.

\subsection{Magnetic properties}

Figures 4(a) and (b) show the magnetization loops of $\mathrm{Fe}_{3} \mathrm{O}_{4}$ nanowires for different diameters (60 nm and above $150 \mathrm{~nm}$, respectively) measured at 3 and $300 \mathrm{~K}$ for parallel magnetic fields. 

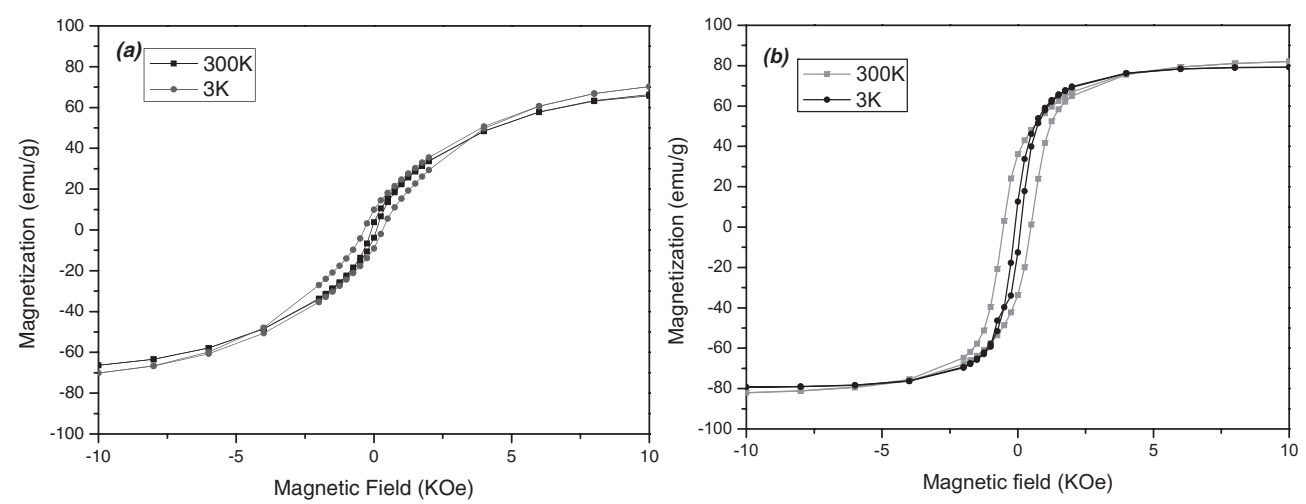

Figure 4. Hysteresis loops at 3 and $300 \mathrm{~K}$ for (a) $60 \mathrm{~nm}$ and (b) above $150 \mathrm{~nm} \mathrm{Fe} \mathrm{O}_{4}$.

Changing the magnetic field orientation induces no modification of the magnetization. The $\mathrm{Fe}_{3} \mathrm{O}_{4}$ nanowires with a diameter above $150 \mathrm{~nm}$ show a different shape in the hysteresis loop compared to the $60 \mathrm{~nm} \mathrm{Fe}{ }_{3} \mathrm{O}_{4}$ nanowire samples. Typically, the hysteresis obtained for nanowires above $150 \mathrm{~nm}$ quickly reaches saturation at low field. For the $60 \mathrm{~nm}$ diameter nanowires, the magnetization does not saturate up to $\mathrm{MH}=50 \mathrm{kOe}$ and the value at $\mathrm{MH}=10 \mathrm{kOe}$ is about 0.85 times $\mathrm{MH}=50 \mathrm{kOe}(3 \mathrm{~K})$. Moreover, the magnetization values are only slightly smaller than the bulk saturation magnetization at $0 \mathrm{~K}\left(95 \mathrm{emu} \mathrm{g}^{-1}\right)$ whatever the nanowire size. We can note that the coercitivity $\left(H_{\mathrm{c}}\right)$ decreases with the diameter of the nanoparticles. Magnetization measurements as a function of temperature are performed according to the zero-field-cooling (ZFC)-field-cooling (FC) procedure. $M_{\mathrm{ZFC}}$ was measured on warming from 3 to $300 \mathrm{~K}$, whereas $M_{\mathrm{FC}}$ was recorded during the subsequent cooling. Figures 5(a) and (b) show the results obtained for nanowires with different sizes. For measurements at $H_{\text {appl }}<750 \mathrm{Oe}$, an irreversible magnetic behaviour (a difference between $M_{\mathrm{FC}}$ and $\left.M_{\mathrm{ZFC}}\right)$ is observed over the whole temperature range.

Let us now consider the $\left[-\mathrm{d}\left(M_{\mathrm{FC}}-M_{\mathrm{ZFC}}\right) / \mathrm{d} T\right]$ curves (figure 5(c)). It is worth recalling that, in the presence of independent relaxation phenomena (i.e. non-interacting particles), the temperature derivative of the remnant magnetization reflects the effective distribution of anisotropy energy barriers of the system [27]. A common feature of all theses curves is the presence of two peaks more or less well defined with the applied field-a small peak $T_{\mathrm{b}}$ around the Verwey transition $(\sim 120 \mathrm{~K})$ arising out of an order-disorder transformation. More specifically, below this transition temperature, the cations on the octahedral sites start getting ordered, thus bringing about a lowering of the magnetization (see figures 5(a) and (b)). A second peak at around $T_{\mathrm{a}} \sim 25 \mathrm{~K}$ is consistent with the hypothesized picture of a frozen, disordered magnetic state [28] which does not depend significantly on the magnetic field. This is also confirmed by the lack of magnetization relaxation below $T_{\mathrm{a}} \sim 25 \mathrm{~K}$, as shown in figure 5(d). Below $T_{\mathrm{a}}$, the $\mathrm{Fe}_{3} \mathrm{O}_{4}$ moments do not relax and are frozen in the spin-glasslike state. On increasing the temperature above $T_{\mathrm{a}}$, such a completely frozen state evolves into a regime where the $\mathrm{Fe}_{3} \mathrm{O}_{4}$ becomes progressively unfrozen, according to the distribution of effective anisotropy energy barriers determined by the size and by the strength of the magnetic interaction with the surroundings. On this basis, we can conveniently describe our system as being constituted by two different components: a non-relaxing (quasistatic) ferromagnetic component and a relaxing spin surface corresponding to the amorphous surface surrounding the grains. In other words, it can be assumed that the shell of the nanoparticles is characterized by a random magnetic anisotropy. 

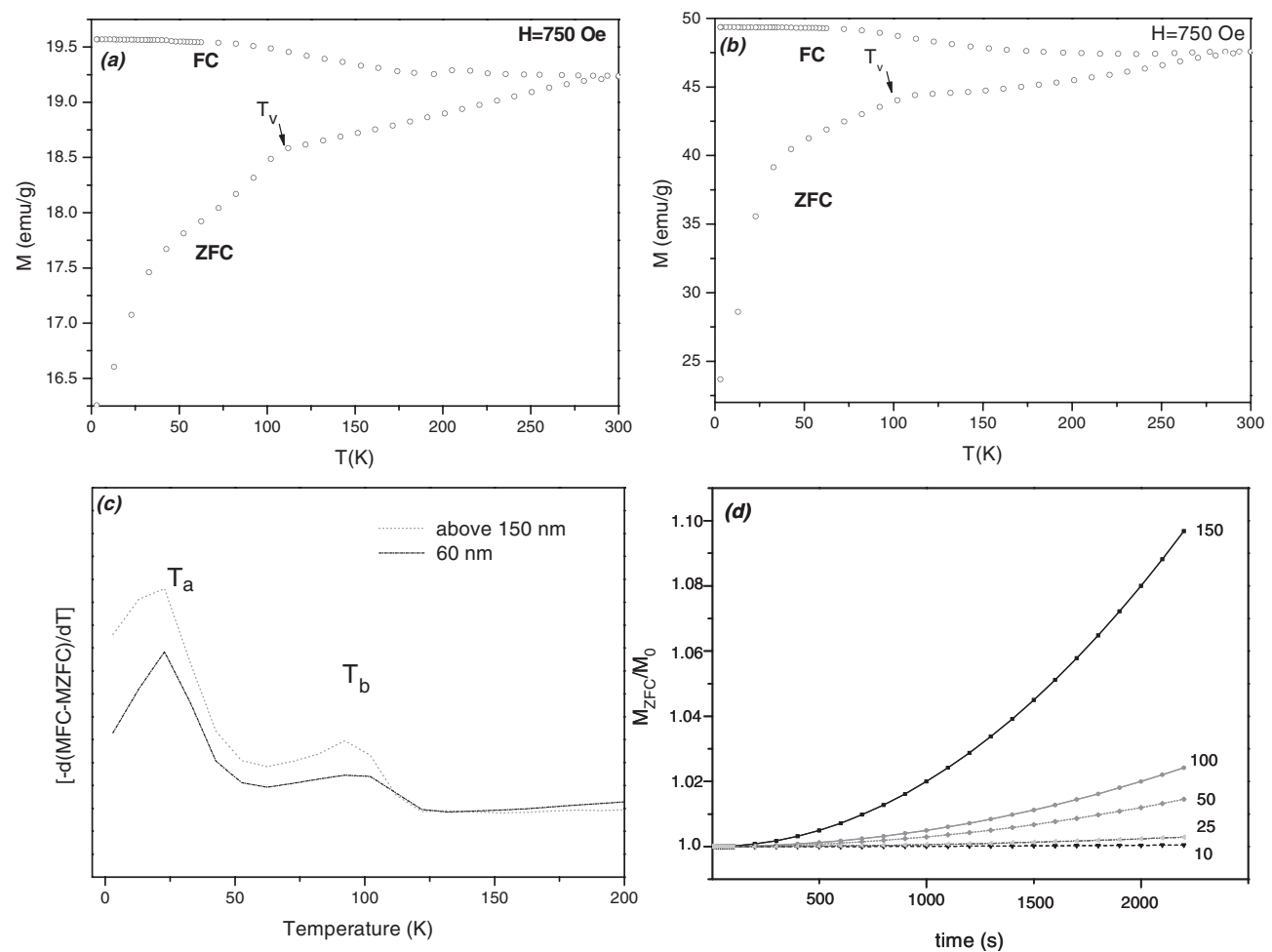

Figure 5. Zero-field-cooling (ZFC, lower branch of each curve) and field-cooling (FC, upper branch) magnetizations $(M)$ as a function of temperature measured on (a) $60 \mathrm{~nm}$ and (b) above $150 \mathrm{~nm} \mathrm{Fe}{ }_{3} \mathrm{O}_{4} \mathrm{NWs}$; (c) temperature derivative $\left[-\mathrm{d}\left(M_{\mathrm{FC}}-M_{\mathrm{ZFC}}\right) / \mathrm{d} T\right]$ of the difference between field-cooled and zero-field-cooled magnetizations measurements; (d) $M_{\mathrm{ZFC}}$ as a function of time measured on $\mathrm{Fe}_{3} \mathrm{O}_{4}(60 \mathrm{~nm}) \mathrm{NWs}$ for $H_{\text {appl }}=100 \mathrm{Oe}$ at the indicated temperatures. $M_{0}$ is the magnetization at the beginning of the measurements.

\subsection{Magnetotransport properties}

Figures 6 and 7 show the longitudinal magnetoresistance $\left(\mathrm{MR}=\left(\rho_{\mathrm{h}}-\rho_{0}\right) /\left(\rho_{0}\right)\right)$ of $\mathrm{Fe}_{3} \mathrm{O}_{4}$ nanowires (for diameters above 150 and $60 \mathrm{~nm}$, respectively) performed at different temperatures.

The MR ratio (around 7\%) observed at room temperature for nanowire diameters above $150 \mathrm{~nm}$ is the highest value of $\mathrm{Fe}_{3} \mathrm{O}_{4}$ reported at $8 \mathrm{kOe}$. Figure 6(b) shows the MR ratio of $\mathrm{Fe}_{3} \mathrm{O}_{4}$ nanowires measured at various temperatures. The MR ratio increases monotonically with decreasing temperature up to a maximum value at around $120 \mathrm{~K}$, and then for temperatures below $90 \mathrm{~K}$ a clear decrease (from $12.5 \%$ at $120 \mathrm{~K}$ to $10.3 \%$ at $50 \mathrm{~K}$ ) is observed. The MR curves (figure 6(a)) show exponential decreases with the magnetic field, and a saturation trend. The maximum MR occurs near the coercive field because of the randomly oriented grains. This MR behaviour obeys the relationship MR $-\left(M / M_{\mathrm{s}}\right)^{2}$ (figure 6(a)) for a non-interacting granular system. If no interaction occurred between the grains, the tunnelling conductance between adjacent grains would be proportional to $\left\langle\cos \phi_{i, j}\right\rangle$, where $\phi_{i, j}$ is the angle between the magnetization directions of grains $i$ and $j$. The averaging over the grains can be expressed by $\mathrm{MR} \propto\left\langle\cos \left(\phi_{i, j}\right)\right\rangle\langle\cos (\theta)\rangle \propto-\left(M / M_{\mathrm{s}}\right)^{2}$, where $\theta$ is the angle between the magnetization directions of the nanoparticles [29, 30]. 

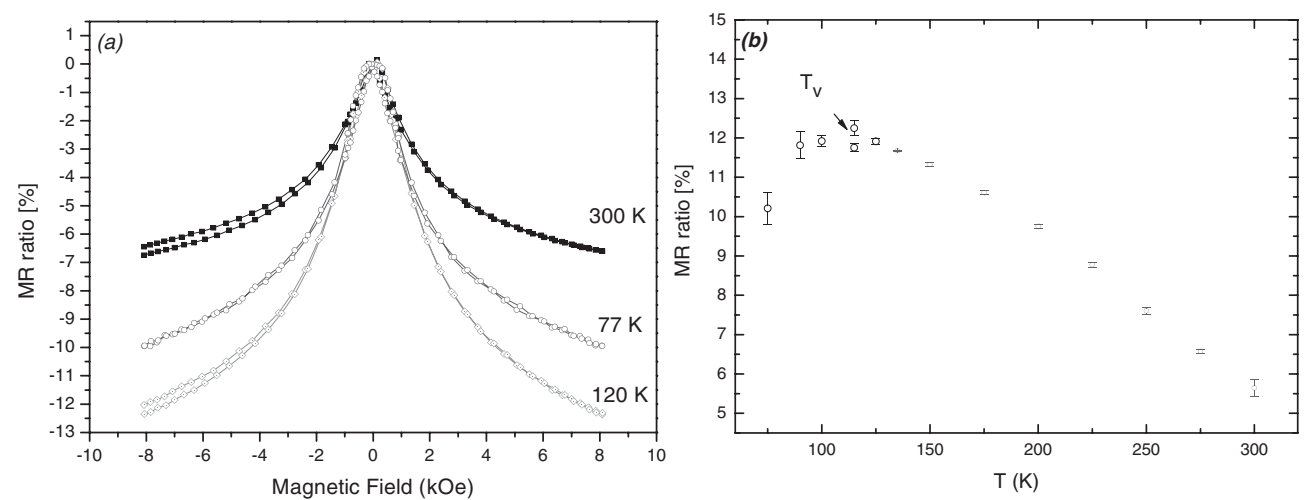

Figure 6. MR for magnetite NW's at different temperature (a) above $150 \mathrm{~nm}$ (b) temperature dependence of the MR at $8 \mathrm{kOe}$.

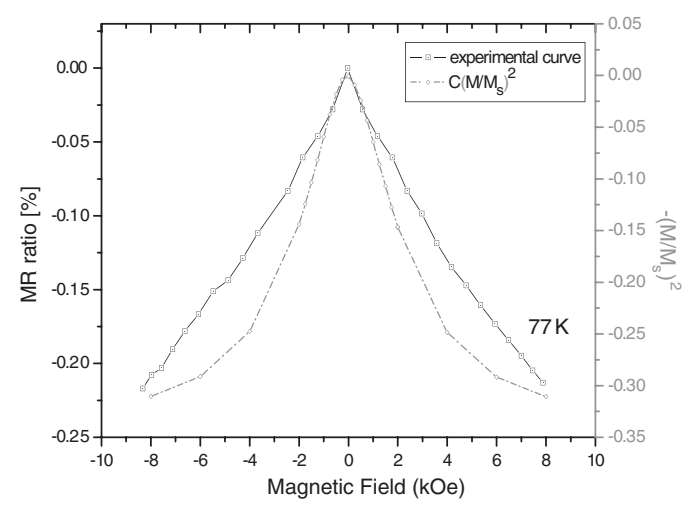

Figure 7. MR for magnetite NWs at $77 \mathrm{~K}$ for $60 \mathrm{~nm}$ and the $-\left(M / M_{\mathrm{S}}\right)^{2}$ curve versus applied field.

In contrary, for the $60 \mathrm{~nm}$ diameter nanowires (figure 7), we did not observe a magnetoresistance at room temperature and only a small magnetoresistance at $77 \mathrm{~K}$ (around $0.2 \%$ ). Here, MR curves show a quasi-linear dependence with applied magnetic field up to $8 \mathrm{kOe}$. A similar MR behaviour has been reported for $\mathrm{Fe}_{3} \mathrm{O}_{4}$ thin film (17 nm) [25] and for the core-shell $\mathrm{Fe}-\mathrm{Fe}$ oxide system where the core was of $\mathrm{Fe}$ and the shell was of Fe oxide [31]. Moreover, as the diameter decreases, the deviation increases between the experimental MR and the calculated MR curves of the non-interacting grains. Such behaviour can be explained by the fact that large nanoparticles have a more uniform magnetization than the smaller grains. Effectively, the quality and the grain size in the $\mathrm{Fe}_{3} \mathrm{O}_{4}$ nanowires will depend strongly of the diameter of the nanotemplates. It is found that the crystallinity of small $\mathrm{Fe}_{3} \mathrm{O}_{4}$ nanowires is relatively poor, with a significant amorphous phase. In the case of small nanowires (small grains), the surface spin disorder phase in the grain boundaries will be more predominant. In summary, this study of transport properties carried out on $\mathrm{Fe}_{3} \mathrm{O}_{4}$ nanowires with different sizes and morphologies suggests that the large negative MR observed for magnetite nanowires above $150 \mathrm{~nm}$ in diameter can be accounted for by spin-dependent tunnelling through a network of contiguous nanoparticles. In zero magnetic fields, the magnetization $(M)$ of each nanoparticle is oriented randomly, inducing a spin-disordered state. The application of an external magnetic field aligns all the magnetic moments and reduces the spin disorder in the case of large grains. 
Consequently, there is an increase in the spin-dependent tunnelling probability, which leads to a reduction in resistance. When the particles size decreases (nanowires of $60 \mathrm{~nm}$ ), the surface effect is more and more important and affects the magnetotransport properties.

\section{Conclusion}

We have studied two types of nanostructured magnetite produced by electrodeposition. Brightfield images of electron microscopy showed contiguous well-bound nanoparticles for $\mathrm{Fe}_{3} \mathrm{O}_{4}$ nanowires with diameters above $150 \mathrm{~nm}$. Smaller nanowires (of around $60 \mathrm{~nm}$ ) exhibit a polycrystalline structure. The stoichiometry of the $\mathrm{Fe}_{3} \mathrm{O}_{4}$ phase has been investigated by Mössbauer spectroscopy and thermoelectrical measurements, which definitively confirm the formation of $\mathrm{Fe}_{3} \mathrm{O}_{4}$ in a slight non-stoichiometric phase. Zero-field cooled measurements show evidence of the Verwey transition, which is also seen in the temperature dependence of the MR. The largest nanowires have the characteristics of a granular system: small saturation field and large magnetoresistance. The smallest nanowires do not saturate at $8 \mathrm{kOe}$, and have a small magnetoresistance which does not correlate to the magnetization, as in a granular system. Effectively, in this case we expect that the magnetoresistance is affected by the surface spin disorder.

\section{References}

[1] Baibich M N, Broto J M, Fert A, Nguyen van Dau F, Petroff F, Etienne P, Creuzet G, Friedrich A and Chazelas J 1988 Phys. Rev. Lett. 612472

[2] Binash G, Grunberg P, Saurenbach F and Zinn W 1989 Phys. Rev. B 394828

[3] Coey J M D, Berkowitz A E, Balcells L, Putris F F and Parker F T 1998 Appl. Phys. Lett. 72734

[4] Dowben P A and Skomski R 2003 J. Appl. Phys. 937948

[5] Eerenstein W, Palstra T T M, Hibma T and Celotto S 2003 Phys. Rev. B 68014428

[6] Eerenstein W, Palstra T T M, Hibma T and Celotto S 2002 Phys. Rev. B 88247204

[7] Seneor P, Fert A, Montaigne F, Petroff F and Vaurs A 1999 Appl. Phys. Lett. 744017

[8] Ziese M and Blythe H J 2000 J. Phys.: Condens. Matter 1213

[9] Margulies D T, Parker F T, Rudee M L, Spada F E, Chapman J N, Aitchison P R and Berkowitz A E 1997 Phys. Rev. B 795162

[10] Terrier C, Abid M, Arm C, Serrano-Guisan S, Gravier L and Ansermet J-Ph 2005 J. Appl. Phys. 98086102

[11] Gravier L, Fàbian A, Rudolf A, Cahin A, Wegrove J E and Ansermet J-Ph 2004 Meas. Sci. Technol. 15420

[12] Bauminger R, Cohen S G, Marinov A, Ofer S and Segal E 1961 Phys. Rev. 1221447

[13] Fujii T, Takano M, Katano R, Bando Y and Isozumi Y 1989 J. Appl. Phys. 663168

[14] Fujii T, Takano M, Katano R, Bando Y and Isozumi Y 1990 J. Cryst. Growth 99606

[15] Daniels J M and Rosenswaig A 1969 J. Phys. Chem. Solids 301561

[16] Constantin C and Rosenberg M 1971 Solid. State Commun. 9675

[17] Kuipers A J M and Brabers V A M 1976 Phys. Rev. B 141401

[18] Lavine J M 1959 Phys. Rev. 114482

[19] Griffiths B A, Elwell D and Parker R 1970 Phil. Mag. 22163

[20] Gong G Q, Gupta A, Xiao G, Qian W and Dravid V P 1997 Phys. Rev. B 565096

[21] Sheng P 1980 Phys. Rev. B 212180

[22] Sondheimer E H 1952 Adv. Phys. 11

[23] Liu H, Jiang E Y, Bai H L, Zheng R K and Zhang X X 2003 J. Phys. D: Appl. Phys. 362950

[24] Verwey E J W 1939 Nature 144327

[25] Park C, Peng Y, Zhu J G, Laughlin D E and White R M 2005 J. Appl. Phys. 97 10C303

[26] Kneller E F and Luberosky 1963 J. Appl. Phys. 34656

[27] O'Grady K and Chantrell R W 1992 Magnetic Properties of Fine Particles ed J L Dormann and D Fiorani (Amsterdam: North-Holland) p 93

[28] Del Bianco L A J M and Brabers V A M 2002 Phys. Rev. B 66174418

[29] Ziese M, Hohne R, Semmelhack H C, Reckentin H, Hong N H and Esquinazi P 2002 Eur. Phys. J. B 28415

[30] Evetts J E, Blamire M G, Mathur N D, Isaac S P, Teo B S, Cohen L F and Macmanus-Driscoll J L 1998 Trans. R. Soc. Lond. A 3561593

[31] Savini L 2002 J. Appl. Phys. 918593 